\title{
Epoxy Networks Reinforced with Polyhedral Oligomeric Silsesquioxanes: Structure and Segmental Dynamics as Studied by Solid-State NMR
}

\author{
Jiri Brus*, Martina Urbanová, and Adam Strachota
}

Supporting information

In this section a theoretical calculation of ${ }^{1} \mathrm{H}^{13} \mathrm{C}$ dipolar spectra of small-size spin clusters based on time independent average Hamiltonian theory is presented. The results are compared with dipolar profiles obtained by $2 \mathrm{D}$ experiments based on Lee-Goldburg off-resonance crosspolarization (LG-CP).

\section{Simulation}

The simulation of ${ }^{13} \mathrm{C}-\left\{{ }^{1} \mathrm{H}\right\}$ dipolar spectra were performed using FORTRAN programs written by us previously (Brus J. et al. Solid State Nuclear Magnetic Resonance 2005, 27, 180). The programs calculate exact ${ }^{13} \mathrm{C}$ frequencies which are modulated by dipolar couplings with neighboring ${ }^{1} \mathrm{H}$ spins for every crystallite orientation. Powder averaging was performed letting the rotation axis to move around the full space angle. At numerical integration, $64 \mathrm{~K}$ points were used with both azimutal and polar angles for the interval from $0^{\circ}$ to $90^{\circ}$. The azimutal angle runs from $0^{\circ}$ to $90^{\circ}$ for more symmetric moieties and to $180^{\circ}$ for less symmetric ones; the polar angle runs $0^{\circ}$ to $90^{\circ}$ in all cases. This results to $4 \mathrm{G}$ or $8 \mathrm{G}$ integration points together. $2 \mathrm{~K}$ spectral points on unit interval were calculated. A few spectra were calculated with two different orientation of the moiety with respect to coordinate axis system; the comparison of the two results shows that the used integration grid with the abovementioned spectral resolution yields mostly four significant digits correct, which is fully sufficient for a comparison with experimental results. No artificial broadening was applied in order to analyze calculated dipolar spectra adequately.

The dipolar spectra were simulated for wide range of local geometries of several spin systems. For the three-spin system the angle $\alpha$ was varied between $180^{\circ}$ and $90^{\circ}$. It should be noted that the dipolar spectra calculated for the angle $\alpha$ complementary to $180^{\circ}$ are identical. In all cases the intenuclear distance $r_{\mathrm{CH} 1}$ was kept constant while $r_{\mathrm{CH} 2}$ was ranging from $r_{\mathrm{CH} 2}=$ $r_{\mathrm{CH} 1}$ to $r_{\mathrm{CH} 2}=2 r_{\mathrm{CH} 1}$. For every angle $\alpha$ dipolar spectra were simulated for thirteen arrangements of spins with variable intenuclear distance $r_{\mathrm{CH} 2}$. The distance $r_{\mathrm{CH} 2}$ is related to $r_{\mathrm{CH} 1}$ by multiplication factor $2^{\frac{n}{12}}(n=0,1, \ldots, 12)$.

\section{Results}

In the dipolar spectra of three-spin systems usually several singularities and jumps arise, and the formation of singularities which splitting is equal to the effective dipolar coupling constant for isolated-like spin pairs is not general (Figure S1). These maxima appear only if the angle between internuclear vectors varies between $45^{\circ}$ and $135^{\circ}$. However, it is also demonstrated that for a typical $\mathrm{CH}_{2}$ group with $\mathrm{H}-\mathrm{C}-\mathrm{H}$ bond angle $109^{\circ}$ the simplified isolated-like spin-pair approach for the analysis of dipolar spectra is still valid because the splitting between the main maxima (singularities) in the dipolar spectrum directly reflects effective dipolar coupling constant that is expected for an isolated spin pair. This finding is confirmed by the comparison with experimentally determined dipolar spectra for $\mathrm{CH}$ and $\mathrm{CH}_{2}$ groups (Figure S2). 
Further the influence of additional more remote spin was tested (Figure S3). As an example we chose simple three-spin system with constant $\mathrm{H}-\mathrm{C}-\mathrm{H}$ bond angle $109^{\circ}$ and variable interatomic distance $r_{\mathrm{CH} 2}=r_{\mathrm{CH} 1} \ldots .2 * r_{\mathrm{CH} 1}$. We must admit that in some cases additional internal singularities can be detected and internuclear distances between carbon and both close and remote protons can be determined. However, if the distance of the remote proton is two-times longer than the distance of the close one, the overall effect on the shape of dipolar spectrum is almost negligible. This situation is encountered in typical organic solids (aliphatic chains). The distance of the nearest proton form carbon is ca. 1.09A (one-bond spin pair) while the distance of the next neighboring proton is about 2.13A. Consequently it is clear that the effect of remote protons on the pattern of dipolar spectra rapidly decreases with increasing internuclear distance. From this follows that the presence of additional remote protons in the system has only negligible effect on the dipolar profiles of $\mathrm{CH}$ and $\mathrm{CH}_{2}$ groups. This trend is independent on $\mathrm{H}-\mathrm{C}-\mathrm{H}$ angle (Figure S4 and S5). Such finding confirms that dipolar profiles of $\mathrm{CH}$ and $\mathrm{CH}_{2}$ groups are dominated by one-bond dipolar couplings and multi-spin behavior can be neglected. 


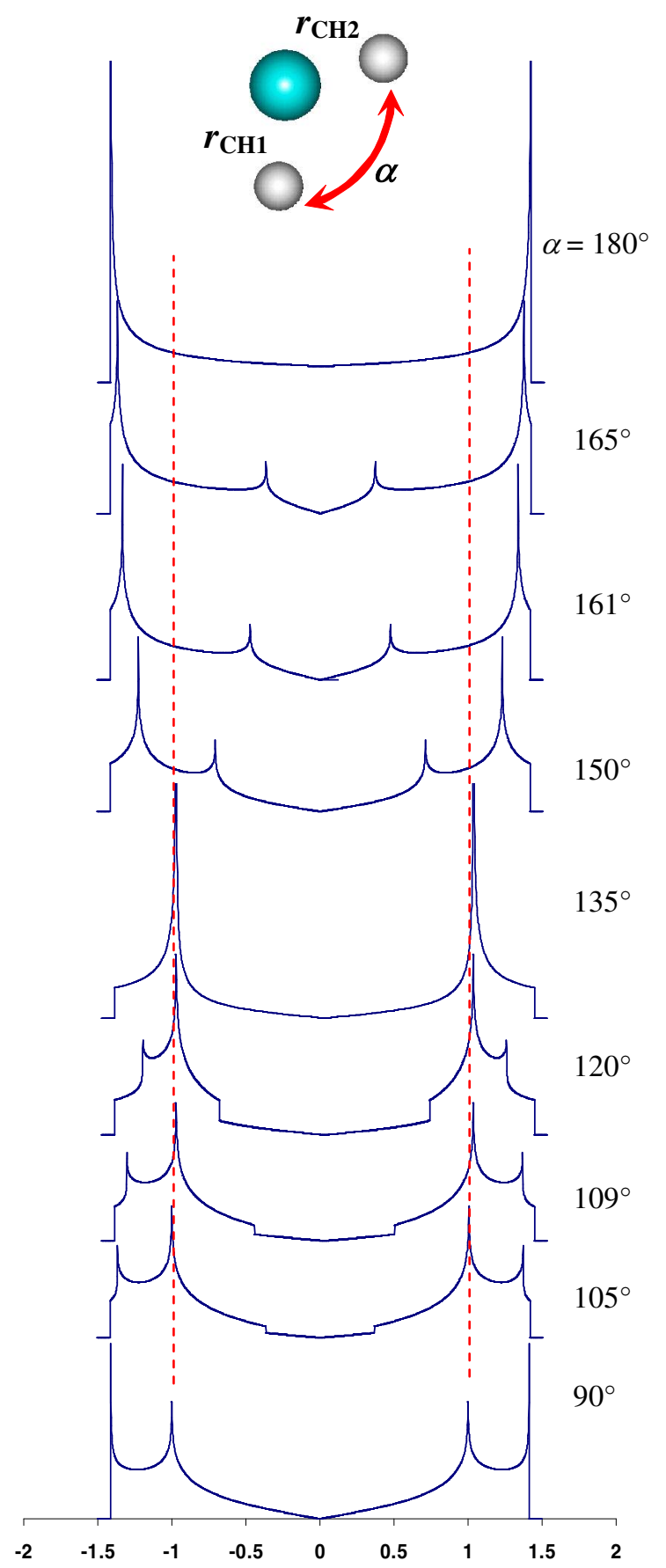

Figure S1. Dipolar spectra calculated for a three spin system $\mathrm{H}(1) \ldots \mathrm{C} \ldots \mathrm{H}(2)$ with constant interatomic distance $r_{\mathrm{CH} 1}=r_{\mathrm{CH} 2}$ and variable angle $\alpha$. The dashed line represents position of maxima (singularities) in the dipolar spectrum calculated for isolated spin pair $\mathrm{C}-\mathrm{H}$ with the same interatomic distance. 


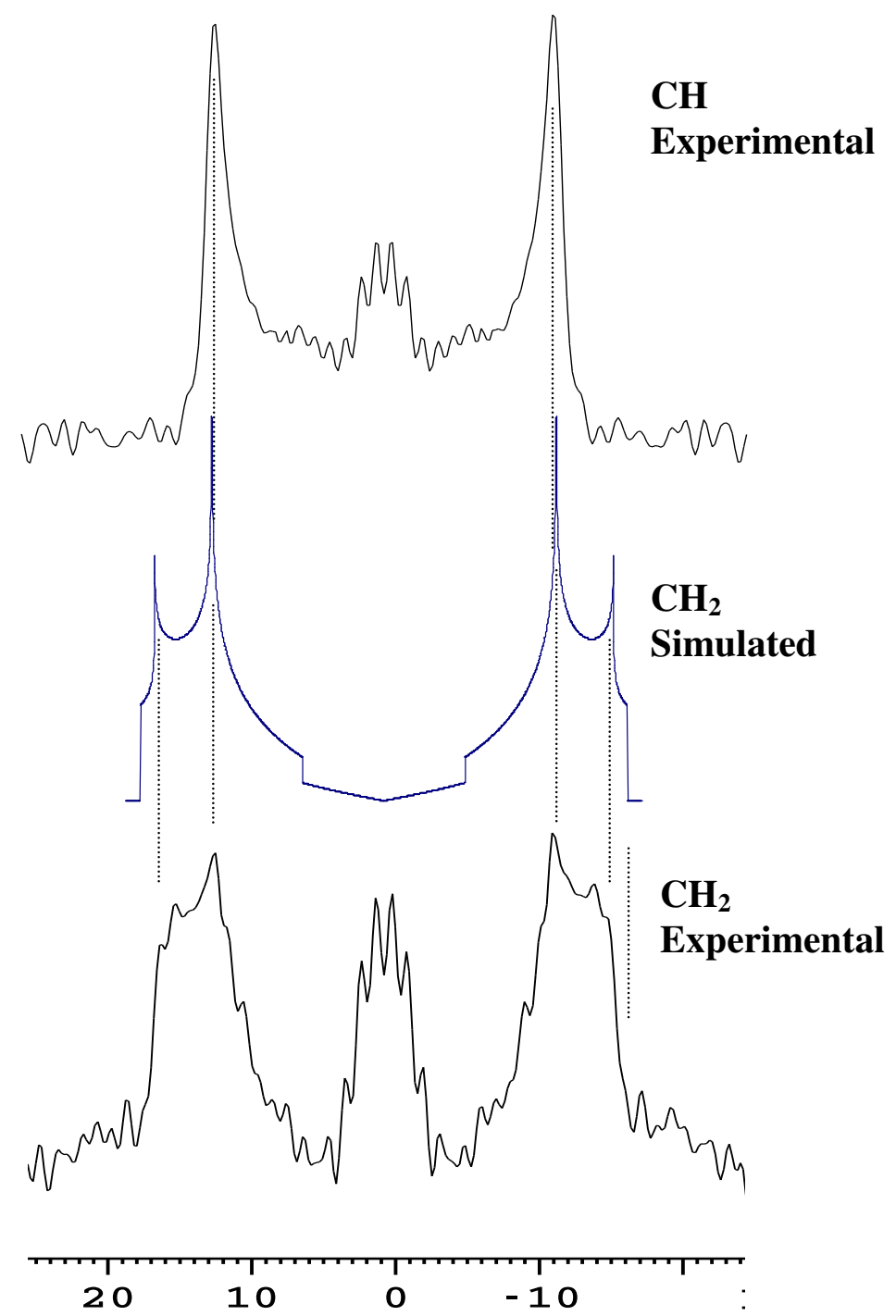

Figure S2. Experimentally determined and simulated dipolar spectra of $\mathrm{CH}_{2}$ and $\mathrm{CH}$ structure units. 


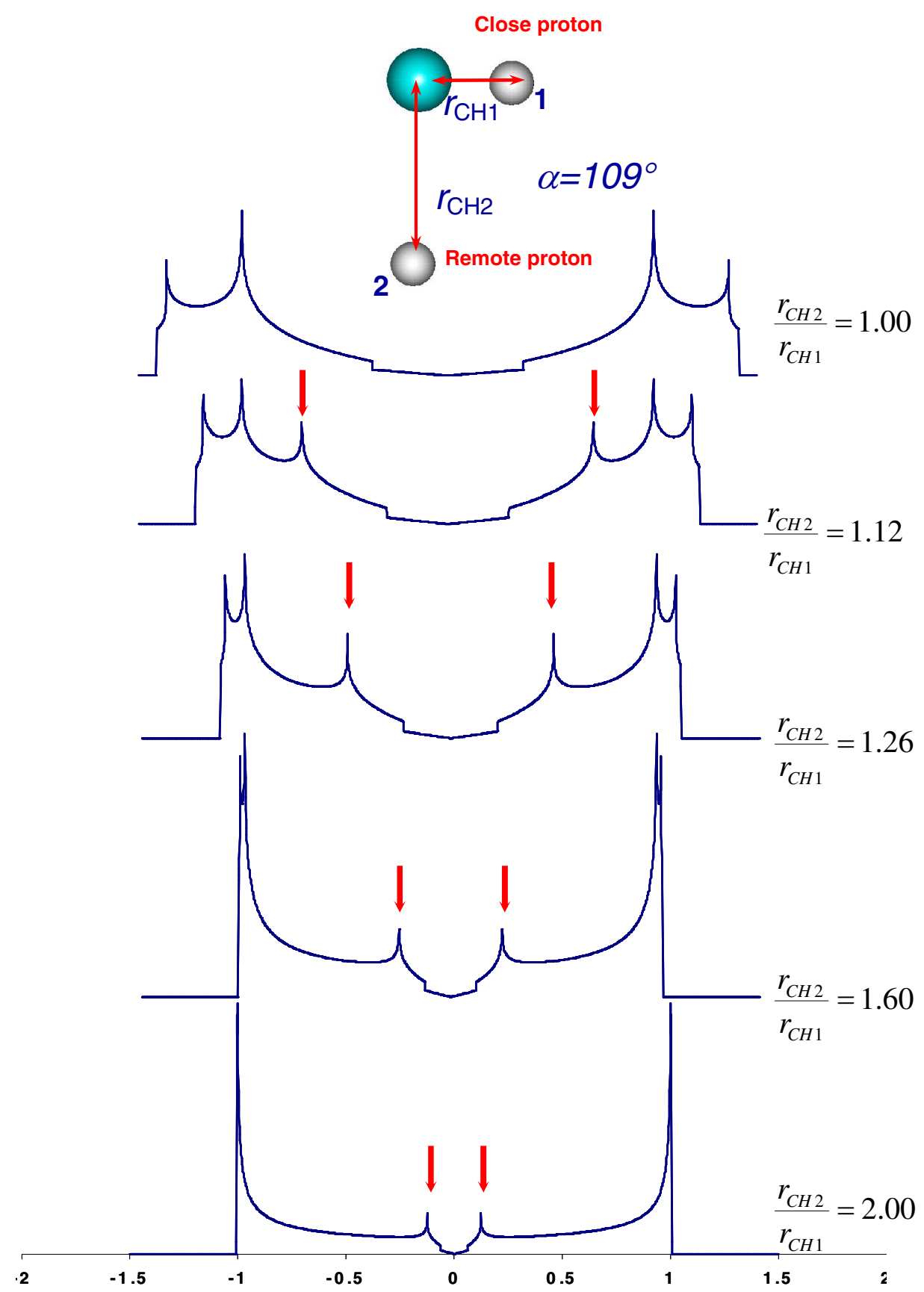

Figure S3. Dipolar spectra simulated for three spin systems with variable angle $\alpha$ and increasing internuclear distance $r_{\mathrm{CH} 2}$. The ratio of interatomic distances $r_{\mathrm{CH} 2} / r_{\mathrm{CH} 1}$ increases from 1 to 2 (from the top to the bottom). The angle $\alpha$ is $109^{\circ}$. Additional singularities reflecting contribution of remote protons are marked by thick arrows. 


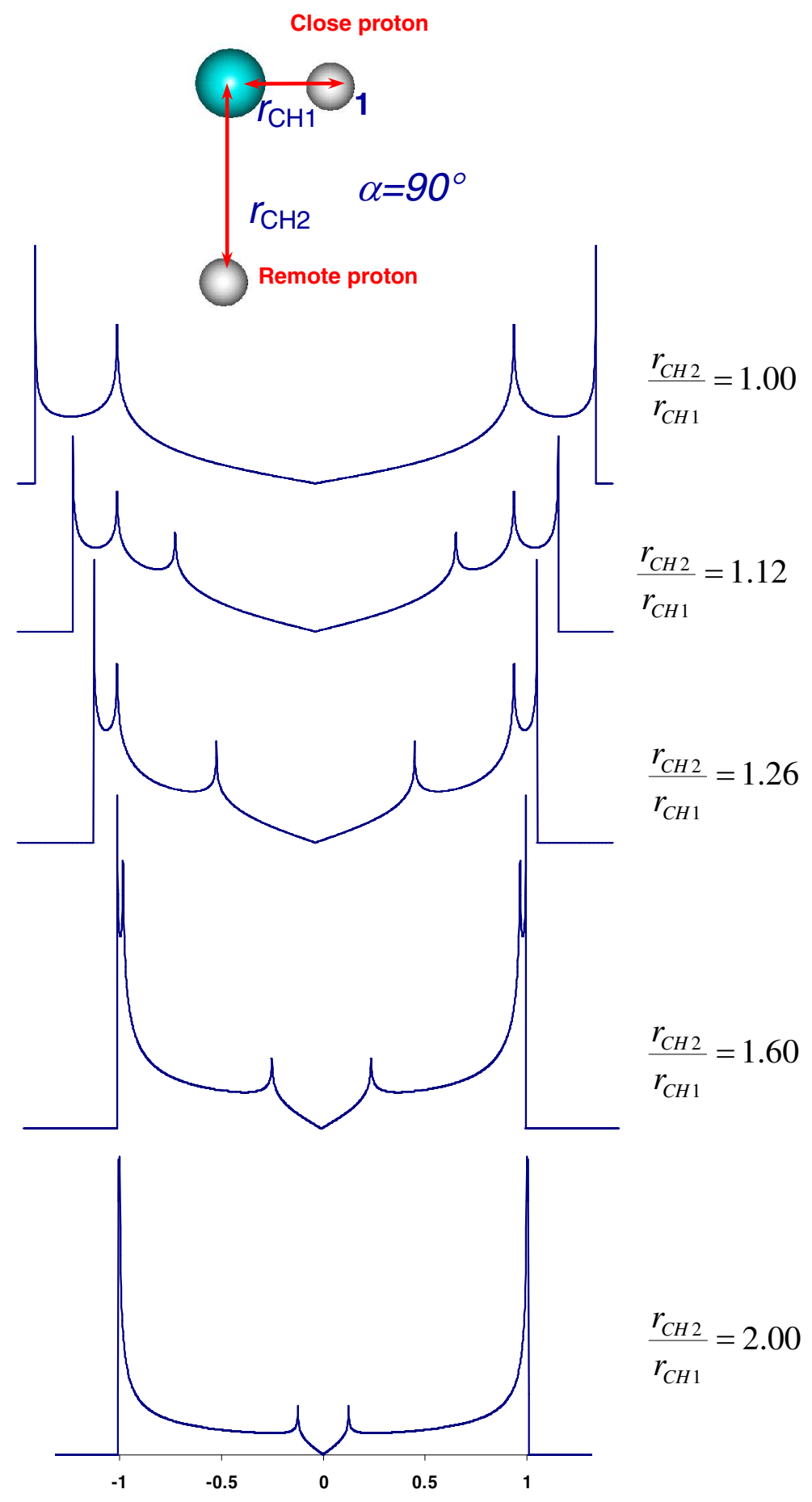

Figure S4. Dipolar spectra simulated for three spin systems with variable angle $\alpha$ and increasing internuclear distance $r_{\mathrm{CH} 2}$. The ratio of interatomic distances $r_{\mathrm{CH} 2} / r_{\mathrm{CH} 1}$ increases from 1 to 2 (from the top to the bottom). The angle $\alpha$ is $90^{\circ}$. 


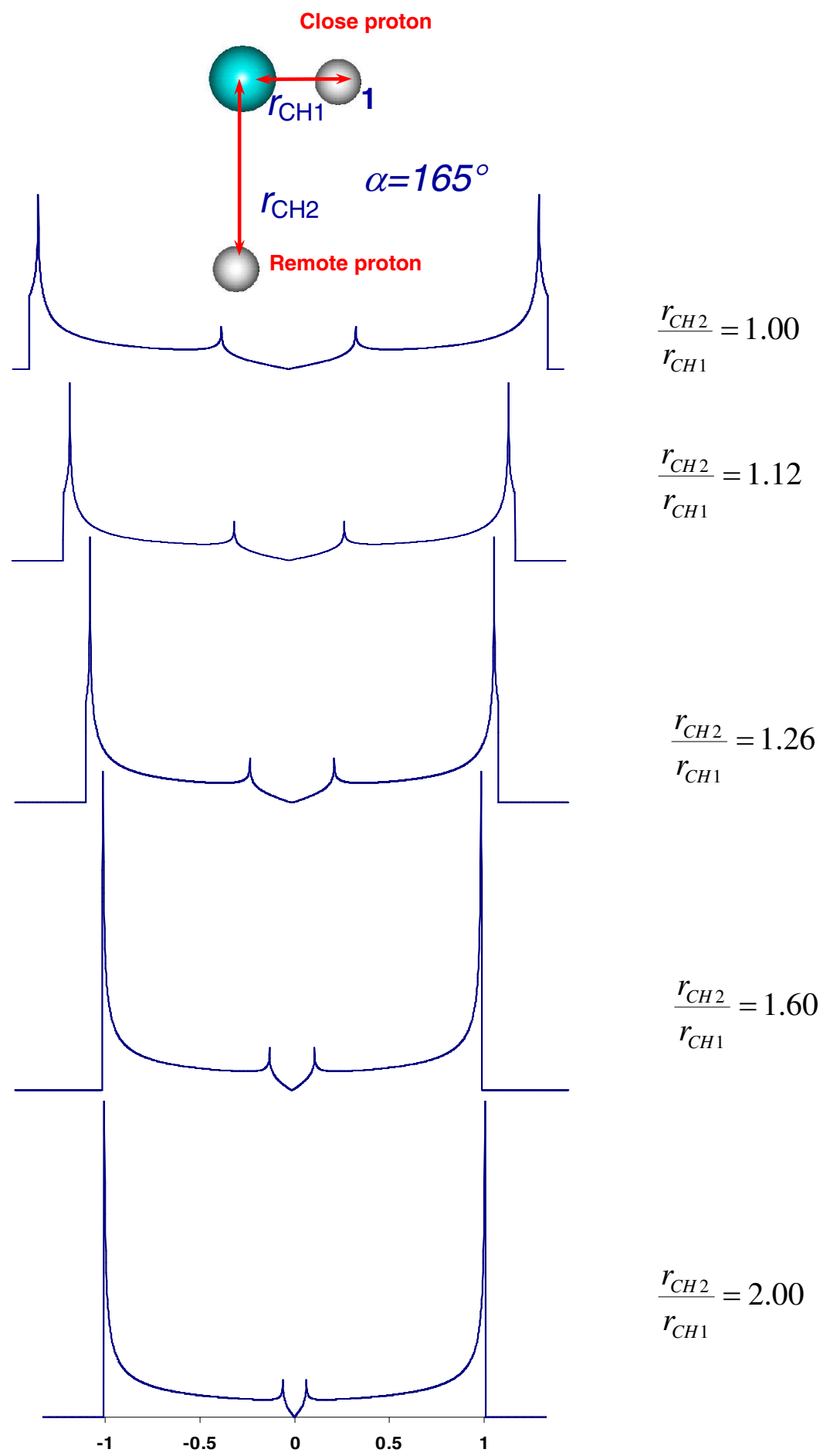

Figure S5. Dipolar spectra simulated for three spin systems with variable angle $\alpha$ and increasing internuclear distance $r_{\mathrm{CH} 2}$. The ratio of interatomic distances $r_{\mathrm{CH} 2} / r_{\mathrm{CH} 1}$ increases from 1 to 2 (from the top to the bottom). The angle $\alpha$ is $165^{\circ}$. 